EOMmUn Communication et organisation

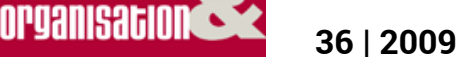

Pour une approche communicationnelle de l'individu au travail

\title{
La reconnaissance au travail par la construction d'une relation agissante
}

La communication des cadres de santé

\section{Valérie Lépine}

\section{OpenEdition}

\section{Journals}

Édition électronique

URL : http://journals.openedition.org/communicationorganisation/941

DOI : 10.4000/communicationorganisation.941

ISSN : 1775-3546

\section{Éditeur}

Presses universitaires de Bordeaux

\section{Édition imprimée}

Date de publication : 1 décembre 2009

Pagination : 96-107

ISBN : 978-2-86781-719-9

ISSN : 1168-5549

Référence électronique

Valérie Lépine, "La reconnaissance au travail par la construction d'une relation agissante », Communication et organisation [En ligne], 36 | 2009, mis en ligne le 14 mars 2011, consulté le 10 décembre 2020. URL : http://journals.openedition.org/communicationorganisation/941 ; DOI : https:// doi.org/10.4000/communicationorganisation.941 
Dossier : Pour une approche communicationnelle de l'individu au travail

\begin{abstract}
Résumé
La reconnaissance, d'abord pensée comme concept philosophique, a fait l'objet de nombreuses analyses psychosociologiques, en particulier pour souligner les attentes des personnels au travail. Cette contribution, inscrite dans la perspective des Sciences de l'Information et de la Communication, vise à examiner le processus même d'élaboration d'une relation où se construit la reconnaissance dans et à travers les pratiques communicationnelles au travail dans le secteur hospitalier. $\mathrm{Si}$ les politiques institutionnelles sur la reconnaissance s'avèrent encore peu visibles en France, les cadres de santé mobilisent, à leur niveau, de nombreuses ressources et compétences communicationnelles pour exprimer de la reconnaissance à l'égard des personnels qu'ils encadrent.
\end{abstract}

\title{
Mots clés :
}

Agir communicationnel, communication au/de travail, cadres de santé, reconnaissance, hôpital

\begin{abstract}
Recognition was early built as a philosophical concept and was analysed from both a psychological and sociological standpoint, in particular so as to stress employee expectations at work. Based on a communicational perspective, this article aims at scrutinizing the process of constructing a relationship out of which recognition emerges through communicative interactions at work in hospitals. Even though in France institutional recognition policies are hardly visible, managers in hospitals are mobilizing numerous communicative competencies so as to express their recognition to their reports.
\end{abstract}

Keywords :

Recognition, communicative action, managers, hospital

Valérie Lé pine est maître de conférences en Sciences de l'Information et de la Communication à l'Université Pierre Mendès France - Grenoble 2, IUT2 département Information Communication. Elle est membre d'ELICO (Equipe de recherche Lyonnaise en Information et COmmunication), EA 4147 - Axe Communication \& Organisations. 


\section{La reconnaissance au travail par la construction d'une relation agissante : la communication des cadres de santé Valérie Lépine}

valerie.lepine@iut2.upmf-grenoble.fr

Conséquences de l'intensification du travail et de l'individualisation accrue des contraintes et des responsabilités (Askenazy et al., 2006) les questions du mal vivre, du stress, de la démotivation ou de la souffrance au travail (Dejours, 1998 ; Loriol, 2000) ont récemment été propulsées dans le débat public. En contrepoint, l'attente de reconnaissance des salariés, pour eux-mêmes, pour leur travail ou pour leur engagement, est devenue une thématique incontournable. Ce concept de reconnaissance d'abord construit par les philosophes, a fait l'objet d'un réinvestissement récent dans le champ des analyses du travail (Dubet, 2006, 2007 ; Lallement, 2007).

Les questionnements précurseurs des philosophes (Honneth, 2000, 2006 ; Ricœur, 2007) posent le problème du fondement même de cette attente, de ce besoin ontologique et social de reconnaissance, dans une perspective éthique et normative; tandis que les sociologues interrogent les processus sociaux par lesquels les différents objets de la reconnaissance (les identités, les groupes sociaux, les métiers, les individus, le travail accompli, l'engagement) sont, ou non, pris en compte par la société. Considérant que la reconnaissance se joue essentiellement dans la qualité des relations qui impliquent les protagonistes des organisations (Brun, Dugas, 2005) nous défendons l'idée qu'une approche par la communication pourrait permettre d'éclairer le processus même d'élaboration discursive de la reconnaissance au travail. En effet, cette dernière repose très concrètement sur des pratiques interactionnelles et énonciatives qui fondent la relation d'où émerge la co-construction d'un sens actant la reconnaissance. La communication en actes et en paroles peut être envisagée comme constitutive d'une relation agissante, produisant de la reconnaissance. La dimension performative des actes de langage (Austin, 1970) faisant reconnaissance intéresse directement le champ des communications organisationnelles dès lors que ces pratiques énonciatives se constituent en normes d'action mobilisant des compétences mais aussi des stratégies communicationnelles.

Nous souhaitons, dans cette contribution, articuler conceptualisation et apports empiriques sur les processus et les dispositifs 
Dossier : Pour une approche communicationnelle de l'individu au travail

communicationnels qui fondent les conditions de réalisation de la reconnaissance en particulier dans le secteur hospitalier. Celui-ci est concerné depuis vingt ans par de profondes réorganisations et a fait l'objet de travaux d'une grande qualité (Grosjean \& Lacoste, 1999), régulièrement renouvelés avec un intérêt particulier pour la catégorie des cadres hospitaliers (Bourret, 2006; Sainseaulieu, 2008). Ces recherches mettent au jour l'importance des interactions quotidiennes dans l'activité et des ajustements multiples nécessaires à l'accomplissement des tâches dans un contexte où la pression subie et le stress des personnels n'ont cessé de s'accentuer.

La recherche que nous avons menée ne porte pas sur le travail hospitalier en tant que tel mais examine plus spécifiquement le rapport qu'une catégorie d'agents - celle des cadres de santé - entretient avec cette question de la reconnaissance dans sa mission d'encadrement. Nous préciserons d'abord le cadre théorique à partir duquel le concept de reconnaissance est mobilisé, puis la méthodologie adoptée afin de rendre compte de la façon dont ces cadres de proximité mobilisent de multiples ressources communicationnelles pour engager un processus de reconnaissance à l'égard des équipes dont ils ont la responsabilité, là où semble-t-il, le soutien de l'institution fait le plus souvent défaut.

Quels apports d'une app roche communicationnelle aux questions de la reconnaissance au travail ?

La problématisation de la reconnaissance du/au travail s'inscrit dans une longue tradition sociologique (Caillé, 2007) autour de la mise en visibilité des luttes, des conflits dans les rapports de production, de classes, depuis l'analyse critique des rapports sociaux jusqu'à l'analyse stratégique des logiques d'acteurs. Les débats ont porté sur la reconnaissance des qualifications de l'homme, du poste de travail ; sur celle des compétences et plus récemment des connaissances. La reconnaissance des collectifs est ainsi explorée sous l'angle des métiers, des professionnalités et des identités au travail (Dubar, 2000). La place et le vécu des individus au travail ont été analysés dans le cadre des approches psychosociologiques développées par l'école des Relations Humaines, poursuivies par les travaux sur la souffrance (Dejours, 1998).

Quel éclairage supplémentaire pourraient apporter les SIC sur la reconnaissance ? En laissant provisoirement de côté ce qui concerne les objets de la reconnaissance, nous tenterons de montrer que les sciences de la communication permettent de mettre l'accent sur le processus communicationnel qui fonde la reconnaissance. En effet, dans sa dimension institutionnelle ou dans les relations quotidiennes 
de travail, elle peut en grande partie se saisir comme (ou à travers) une pratique énonciative, discursive. D'une certaine façon, la reconnaissance se présente comme un processus complexe dans lequel les actes de communication, de nature langagière ou non, occupent un rôle central en raison de leur dimension performative (Austin, 1970).

À partir du cadre théorique proposé par Honneth pour penser la reconnaissance (2004) et en prolongeant quelques-unes de ses hypothèses, nous proposons une confrontation du modèle honnethien aux problématiques exprimées par les cadres de santé. Rappelons très succinctement qu'Honneth distingue trois sphères dans lesquelles se réalisent les principes de reconnaissance dans nos sociétés modernes : la sphère de l'intimité ou encore de l'amour où la reconnaissance se joue dans la relation affective et rend possible l'accès à une identité personnelle et à la confiance en soi ; la sphère du droit où la reconnaissance se fonde sur des droits et devoirs égaux entre les individus permettant d'accéder au respect de soi; enfin, la sphère sociale de la collectivité et de la solidarité dans laquelle la reconnaissance est celle de la contribution singulière de chaque sujet à la société et qui autorise l'accès à l'estime de soi. Le philosophe accorde une place décisive à l'intersubjectivité comme condition formelle de chacune des formes de la reconnaissance et a prêté le flanc à certaines critiques des politologues pointant le risque d'une dérive psychologisante. Ainsi Fraser (2005) a-t-elle mis l'accent sur l'asymétrie des relations sociales et sur les injustices, institutionnalisées dans le cours de l'histoire, des logiques redistributives de reconnaissance, mais aussi des ressources matérielles associées. De son côté, Ferrarese souligne que «si la reconnaissance est un acte performatif qui crée le statut, la définition, le soi qu'il reconnaît, il est le fait de celui qui accorde sa reconnaissance. Dès lors, il y a exercice d'un pouvoir» (Ferrarese, 2005 : 174). Honneth tente de répondre à ces mises en garde en examinant au plus près la question des injustices qu'il choisit d'aborder à partir de l'exploration des «pathologies du social» dans La société du mépris (2006). Il y examine ce qui serait l'envers d'une véritable reconnaissance et formule notamment deux hypothèses qui nous intéressent pour aborder la reconnaissance du point de vue des communications organisationnelles. La première est celle d'une possible déformation ou dénaturation de la reconnaissance lorsqu'elle est mobilisée et instrumentalisée dans le cadre du travail : l'attribution d'éloges pour les qualités ou les compétences des sujets sociaux aurait alors pour "fonction sous-jacente d'intégrer les individus ou groupes sociaux dans l'ordre social dominant $»$ (Honneth, 2006 : 245). Une 
Dossier : Pour une approche communicationnelle de l'individu au travail

seconde hypothèse concerne le déplacement systématique du jugement des qualités de la personne en tant que telle vers l'évaluation de ses compétences hic et nunc, remises en question à l'aune du succès ou de l'échec de chaque «projet». La reconnaissance serait alors continuellement remise en question dans une logique managériale focalisée sur le résultat. Il s'agit de confronter ces hypothèses à la représentation que les cadres de santé se font des pratiques concrètes de la reconnaissance dans leurs unités de soin d'une part, à un niveau institutionnel d'autre part.

\section{Une enquête exploratoire qualitative}

Cette réflexion s'appuie sur une recherche exploratoire auprès de cadres de santé en situation de management opérationnel menée sur la base d'entretiens approfondis et d'une collecte de documents institutionnels (organigrammes, fiches de poste, fiches d'évaluation). Les entretiens ${ }^{42}$ ont été conduits dans dix unités de soin de CHU en Rhône-Alpes et PACA représentant, sans exhaustivité, différentes spécialités médicales (gériatrie, psychiatrie, gynécologie obstétrique, maladies aiguës, chirurgie). Ils ont donné lieu à une analyse verticale des représentations individuelles sur le métier, la fonction, les pratiques professionnelles et à une analyse de contenu horizontale et thématique des tendances plus transversales. Ce sont donc les discours des acteurs - auxquels il est fait crédit d'une certaine capacité réflexive - qui sont analysés et non les interactions langagières au travail.

Lorsqu'on aborde avec ces cadres la question du travail de management, la reconnaissance n'est pas une catégorie évoquée spontanément. Ils parlent d'abord du rôle d'organisation qui «permet à l'équipe de soignants de faire son travail correctement» et se décline en une multitude de tâches visant à remplir ce qui est davantage perçu comme une fonction qu'un métier. Les termes d'animation d'équipe, de valorisation, de motivation adviennent avant celui de reconnaissance, exploré sur relance ou par reformulation dans les entretiens. Cela tend à démontrer que cette notion n'est pas directement conscientisée comme norme d'action professionnelle et lorsqu'elle apparaît, c'est du côté du manque, du sentiment d'injustice ou d'empêchement de la capacité de décider et d'agir (Dubet, 2006).

\footnotetext{
${ }^{42}$ La présente contribution repose sur l'exploitation de dix entretiens conduits entre avril 2008 et mars 2009 auprès de cadres de santé. Cet échantillon permet de poser des pistes d'analyse sans atteindre le seuil de saturation des données.
} 
Pourtant la reconnaissance est partout identifiable dans le récit des cadres de santé. Elle se saisit à travers des actes de communication qui correspondent à des moments, des pratiques, que nous regrouperons en trois grandes catégories: celle de l'activité communicationnelle ordinaire du travail ; celle des moments de représentation et de mise en scène de la reconnaissance; enfin celle des discours et des signes institutionnels.

\section{La reconnaissance au quotidien : dans l'être-là et au fil du dire}

Les manifestations ordinaires de la reconnaissance remplissent une fonction phatique (Jakobson, 1963) : les cadres de santé nouent par leur acte de présence un lien étroit avec l'équipe soignante. Être présent au moment des relèves ne répond pas seulement à une utilité organisationnelle (faire le point, anticiper le travail de la journée, etc.) mais signifie que le cadre est solidairement impliqué avec l'équipe dans le projet de soin des patients. Il assume alors le rôle d'un «passeur de parole » entre les différents acteurs du service : "L'autre jour un médecin est rentré de vacances et il a dit "oh, l'unité est calme!" Bon, pour moi ça c'est un signe de reconnaissance, mais pas que pour moi, pour tout le travail de l'équipe, pour tout le monde. Ça veut dire que les patients sont bien accompagnés... voilà, c'est de la reconnaissance mais que je vais transmettre à tout le monde». Ce travail de lien, quoique qualifié d'invisible (Bourret, 2006) est revendiqué par les cadres. Par exemple, ce cadre de santé qui met une photocopie de la lettre d'une famille satisfaite de la prise en charge d'un patient dans le cahier qui sert de guide journalier de l'activité du service ou tel autre qui retranscrit et épingle sur le panneau d'affichage de la salle des soignants les propos positifs des familles ou des médecins. Ainsi, ce sont bien des compétences communicationnelles qui sont mobilisées pour que la reconnaissance exprimée par les uns (familles, médecins), soit entendue par les autres (soignants et auxiliaires). Les manifestations ordinaires de la reconnaissance s'expriment aussi dans l'activité langagière quotidienne, par «des petits mots avec chacun», des paroles gratifiantes, des compliments adressés personnellement dans le cours de l'activité.

Mais tandis qu'Honneth fait l'hypothèse d'une possible déformation de la reconnaissance à des fins stratégiques, les cadres de santé - quant à eux - se gardent de toute flatterie injustifiée : «Quand ce n'est pas vrai, je ne vais pas le dire!»; "Je sais reconnaître leur boulot, je sais les défendre, par contre je sais dire ce qui ne va pas». Ils se défendent du risque d'un management affectif ou manipulateur : « $J e$ 
Dossier : Pour une approche communicationnelle de l'individu au travail

sais aussi récupérer un niveau où je pose les limites à tout le monde, à moi y compris»; «Il y a cinq ans sans la formation, ça m'était difficile parce qu'il $y$ avait de l'affectif dans la relation». L'expérience acquise permet de passer d'une reconnaissance exprimée dans la sphère de l'affectif - "prendre soin de mon équipe » - à celle formulée dans la sphère de la solidarité et des communautés de valeurs, celle du «travail bien fait». Les cadres mesurent l'importance des attentes de reconnaissance - «Ils en ont besoin, c'est essentiel» - et l'on retrouve ici la nature ontologique de l'attente de reconnaissance formulée par Honneth. Mais en tentant d'y répondre avec des ressources principalement symboliques, les cadres de santé ne sont-ils pas à leur insu les porte-parole d'une logique de consolation?

Curieusement, et comme s'ils avaient l'intuition que la reconnaissance dans le cadre du travail peut aussi être prise dans une autre logique que celle d'une intersubjectivité pure exigeant une forme de symétrie entre les sujets, ils se situent eux-mêmes en dehors d'une relation de réciprocité : "On n'a pas une place où on est apprécié. En fait on n'est pas là pour être aimé, hein! ». Cette oblitération de l'attente de reconnaissance pour eux-mêmes est contredite par d'autres recherches montrant que les cadres de santé ressentent un profond malaise et revendiquent aussi de la reconnaissance (Sainsaulieu, 2008). Cette posture pourrait être le symptôme d'un doute sur leurs propres aspirations en tant que cadres dans une organisation où, au niveau opérationnel, les valeurs du soin prévalent encore sur les logiques gestionnaires attachées à leur fonction.

\section{Les entretiens d'appréciation : une scène pour la reconnaissance}

L'entretien annuel d'appréciation (ou d'évaluation) est obligatoire dans les différentes fonctions publiques et existe depuis quelques années dans le monde de l'hôpital. Il est parfois le seul moment formalisé de face à face entre les personnels de l'unité de soin et le cadre de santé et concentre des enjeux complexes. Tout d'abord il est assez frappant de constater que les cadres évacuent largement leur fonction évaluative: la production d'une fiche d'évaluation et l'attribution d'une note ouvrant droit à la prime individuelle sont repoussées à un autre moment, hors de l'interaction. Dans certains établissements où la culture syndicale est forte, la tradition est d'attribuer systématiquement la note maximale (ou a minima ouvrant droit à la prime) ; les cadres de santé expriment ainsi leur solidarité à l'égard d'une revendication, commune à tous les personnels hospitaliers, de revalorisation des rémunérations, situant ici la 
reconnaissance dans la sphère du droit. Cet entretien va être détourné de sa fonction managériale, pour être transformé, du point de vue des cadres, en un véritable moment de reconnaissance. En tentant une analyse communicationnelle de la situation, nous avançons qu'en premier lieu il assume une fonction de méta-communication (Jakobson, 1963) : le cadre et l'agent hospitalier se mettent d'accord sur un cadre d'entente commun (bilan pour l'année écoulée et objectifs pour l'année à venir). En second lieu, il remplit une fonction expressive : c'est un moment d'engagement fort dans la relation professionnelle qui rend possible la rencontre de deux sujets durant laquelle des dimensions plus personnelles peuvent être abordées. Enfin, l'entretien a aussi une fonction conative, car ce dialogue doit déclencher une amélioration de l'action, un engagement du personnel dans de nouveaux projets (formation, groupes de travail, prise de responsabilités...).

À l'initiative des cadres, les entretiens vont être quelquefois scénarisés autour d'une unité de temps (groupés sur quelques semaines); d'une unité de lieu (le bureau fermé du cadre, téléphone coupé); et d'une unité d'action (la fiche de poste sert de base à l'interaction). Il s'agit donc d'un véritable dispositif communicationnel qui place au cœur de l'interaction une scénarisation du processus de reconnaissance des personnels hospitaliers. Concrètement, cette reconnaissance de la personne, de son travail et de son engagement sera actée par l'attribution d'une formation, la désignation de l'agent comme référent d'un domaine du soin (l'alimentation, l'hygiène, etc.), la prise en compte de demandes d'aménagement du planning (dans la mesure des marges de manœuvre, souvent étroites, dont les cadres disposent). Il y a bien-là une éthique de la reconnaissance dans le rapport intersubjectif, qui permet à chacun d'entretenir un rapport positif à soi-même ; mais qui apparaît comme une fragile compensation des insuffisances vécues au niveau de l'institution «les soignants nous disent fréquemment "l'hôpital nous prend pour des pions", qu'ils ne sont pas entendus ».

\section{La reconnaissance, gran de absente d es discours et des docu ments} institutionnels

La troisième catégorie d'actes de communication agissant dans le processus de reconnaissance se situe au niveau de l'institution. Une de nos hypothèses de départ allait dans le sens développé par Honneth (2006) dénonçant la dénaturation de la reconnaissance comme idéologie, autour de l'idée que, dans certaines organisations, la reconnaissance pouvait être utilisée comme un véritable système de 
Dossier : Pour une approche communicationnelle de l'individu au travail

management exprimé par des discours ou des signes organisés en système de communication. Or il ressort majoritairement de l'enquête que les CHU visités n'ont pas de «vision » ou de projet mobilisant explicitement les ressorts de la reconnaissance. Il existe certes des politiques de formation et de gestion des compétences et certains cadres mentionnent un volet social et de ressources humaines dans les projets d'établissement. Mais ces dimensions ne trouvent pas ou peu d'incarnation concrète perçue au sein des unités de soin. Cependant, à partir des documents recueillis, il est difficile de conforter l'hypothèse d'une idéologie de la reconnaissance, identifiable «quand une institution émet des promesses de reconnaissance qu'elle ne peut satisfaire 》 (Renault, 2007 : 132).

Le constat est plutôt celui de la multiplication des indices de nonreconnaissance, notamment des cadres de santé au niveau institutionnel. Les fiches de poste, les organigrammes sont des documents qui assurent une fonction référentielle dans la communication organisationnelle. Or certaines de ces fiches de poste reprennent textuellement les arrêtés relatifs à la formation et aux missions des cadres de santé sans aucune adaptation aux spécificités des postes de travail alors que la prise en charge des patients, la relation aux familles ou encore le travail de coordination de réseaux d'acteurs de santé diffèrent très sensiblement d'un secteur à l'autre. D'autres fiches ont été rédigées en concertation avec les acteurs, mais elles restent vécues comme une forme idéalisée de l'activité concernée, éloignée de la réalité et du poids du travail quotidien. Les missions d'auto-évaluation, de mise en place d'indicateurs de la qualité des soins qui y figurent apparaissent comme des objectifs très difficilement atteignables compte tenu du manque de temps et de visibilité des tâches quotidiennes: "Il y a des jours où je ne pourrais même pas dire ce que j'ai fait mais je n'ai pas arrêté; c'est une quantité de petites choses (...) mais c'est vrai que ça n'a pas de nom $c ̧ a »$. La seconde illustration du défaut de signes de reconnaissance institutionnelle est donnée par les organigrammes, documents formels qui traduisent la vision organisationnelle de la division du travail et de la répartition des responsabilités. L'enquête montre qu'ils peuvent comporter des zones d'ombre : certains organigrammes représentent les rapports hiérarchiques mais aucune des responsabilités de projets ; dans un des services, l'organigramme officiel n'a pas été mis à jour depuis deux ans malgré de nombreux changements. Au final, les cadres de santé sont nombreux à estimer que leur fonction et leur positionnement sont mal représentés dans ces documents. L'attente d'un discours institutionnel fort et audible de reconnaissance en 
direction des cadres du secteur hospitalier a néanmoins récemment suscité une prise de conscience au niveau politique : une mission ${ }^{43}$ a été engagée par le Ministère de la Santé et des Sports pour redéfinir et revaloriser le statut de ces cadres.

\section{Conclusion}

Nous avons tenté de souligner dans cette contribution l'importance des actes de communication adressés par les cadres de santé aux membres de leur équipe dans un processus de reconnaissance par lequel chacun peut "se voir conférer une valeur en tant qu'être de besoin, en tant que sujet autonome doté des mêmes droits que ses semblables, et en tant que sujet capable d'accomplir un certain nombre de choses » (Honneth, 2006: 262). Ces cadres issus de la filière des soignants ont partagé les tâches quotidiennes et les contraintes des agents hospitaliers. Aussi la sincérité des énoncés dispensés dans l'activité langagière quotidienne et les efforts déployés pour privilégier les moments d'interaction où l'expression d'une valeur évaluative positive est possible, ne saurait être rabattue dans un agir stratégique purement instrumental. En reconnaissant la valeur des agents dont ils ont aujourd'hui la responsabilité managériale, c'est à travers eux la valeur de leur contribution passée à cette même place que les cadres reconnaissent aussi. Cependant, cette activité communicationnelle qui noue une relation agissante comme acte de reconnaissance, "reste un acte en quelque sorte incomplet tant qu'il ne débouche pas sur des comportements manifestant effectivement la valeur qu'il exprime » (Honneth, 2006 : 271). Ce travail devrait donc se poursuivre avec la prise en compte de la traduction en dispositions matérielles, juridiques et institutionnelles de la reconnaissance. Ce niveau de l'institution n'a été abordé dans cette étude que du point de vue des traces énonciatives, des documents «exprimant» ou non des formes de reconnaissance. Cette approche comporte une limite quant à sa capacité à produire une critique fondée de l'institution hospitalière, cadre de la production des rapports sociaux. La question des luttes pour la reconnaissance croise nécessairement celle des formes et des rapports de pouvoir. Aussi, nous reprenons à notre compte la critique adressée à Honneth par Renault (2004: 180) à l'égard de ce qu'il appelle le «concept expressif de la reconnaissance» qui correspondrait à une conception des institutions en tant qu'elles «constituent les conditions permettant soit de stabiliser les relations

43 http://www.sante-sports.gouv.fr/hopital/actualites/mission-cadreshospitaliers - dernière consultation le 10/11/09. 
Dossier : Pour une approche communicationnelle de l'individu au travail

de reconnaissance, soit de perpétuer les obstacles à leur développement»; ainsi que sa proposition de «passer d'une théorie des institutions comme expression de la reconnaissance à une théorie des institutions comme constitution des relations de reconnaissance » (2004 : 194). Cela engage à poursuivre le travail de confrontation et d'articulation des dimensions micro, méso et macro-sociales du travail.

\section{Bibliographie}

ASKENAZY P. et al., Coord. Organisation et intensité du travail. Toulouse : Octares, 2006.

BOURRET P. Les cadres de santé à l'hôpital. Un travail de lien invisible. Paris : Seli Arslan, 2006.

AUSTIN J.-L. Quand dire c'est faire. Paris : Seuil, 1962.

BRUN J-P. et DUGAS N. La reconnaissance au travail : analyse d'un concept riche de sens. in : Gestion. HEC Montréal, 2005, vol.30, n²2.

CAILlE A. Vers une théorie de l'action et du sujet. in: Revue $d u$ MAUSS. 2008, $\mathrm{N}^{\circ} 32$, pp. 69-77.

DEJOURS C. Souffrance en France : la banalisation de l'injustice sociale. Paris : Seuil, 1998.

DUBAR C. La crise des identités : l'interprétation d'une mutation. Paris : PUF, 2000.

DUBET F. Injustices. L'expérience des inégalités au travail. Paris : Seuil, 2006.

DUBET F. Injustices et reconnaissance. in : CAILLE A. Dir. La quête de reconnaissance, nouveau phénomène social total. Paris: La Découverte, MAUSS, 2007.

GROSJEAN M., LACOSTE M. Communication et intelligence collective. Le travail à l'hôpital. Paris : PUF, 1999.

FERRARESE E. La reconnaissance, le tort et le pouvoir. in: FRASER N. Qu'est-ce que la justice sociale? Reconnaissance et redistribution. Paris : La Découverte, 2005, pp. 159-177.

HONNETH A. La lutte pour la reconnaissance. Paris : Le Cerf, 2000.

HONNETH A. La société du mépris. Vers une nouvelle Théorie critique. Paris : La Découverte, 2006. 
HONNETH A. Reconnaissance et reproduction sociale. in : PAYET JP. \& BATTEGAY A. Éd. La reconnaissance à l'épreuve. Lille : Presses Universitaires Septentrion, 2008, pp. 45-58.

JAKOBSON R. Essais de linguistique générale. Tome 1, Paris : Ed de Minuit, 1963.

LALLEMENT M. «Qualités du travail et critique de la reconnaissance ». in : CAILLE A. Dir. La quête de reconnaissance, nouveau phénomène social total. Paris: La Découverte, MAUSS, 2007, pp. 71-88.

LORIOL M. Le temps de la fatigue. La gestion du mal être au travail. Paris : Anthropos, 2000.

MISPELBLOM BEYER F. Encadrer, un métier impossible. Paris : Armand Colin, 2006.

RENAULT E. Reconnaissance, institutions, injustice. in : Revue du MAUSS, 2004, n²3, pp. 180-195.

RENAULT E. Reconnaissance et travail. in: Travailler. Revue internationale de Psychopathologie et de Psychodynamique du Travail, 2007, n¹8, pp. 119-135.

RICOEUR P. Parcours de la reconnaissance. Trois études. Paris : Folio Essais, 2007.

SAINSAULIEU I. Dir. Les cadres hospitaliers : représentations et pratiques. Paris : Lamarre, 2008. 